\title{
A conditional sterile mutation eliminates surface components from Arabidopsis pollen and disrupts cell signaling during fertilization
}

\author{
Daphne Preuss, ${ }^{1,3}$ Bertrand Lemieux, ${ }^{2}$ Grace Yen, ${ }^{1}$ and Ronald W. Davis ${ }^{1}$ \\ ${ }^{1}$ Department of Biochemistry, Stanford University, Stanford, California 94305 USA; ${ }^{2}$ Department of Biology, York \\ University, Toronto, Ontario M3J-1P3 Canada.
}

\begin{abstract}
Plants distinguish among the pollen grains that land on the stigma, permitting only compatible pollen to fertilize egg cells. To investigate these cell-cell interactions, Arabidopsis mutations that affect pollen-pistil communication were isolated. A male-sterile mutation that disrupts pollen-pistil interactions by eliminating the extracellular pollen coat (tryphine) is described here. Stigma cells that contact the mutant pollen produce callose, a carbohydrate synthesized in response to foreign pollen. The mutant pollen fails to germinate because it does not absorb water from the stigma, yet germinates in vitro, indicating it is viable. The defect is also conditional; high humidity results in pollen hydration and successful fertilization. Analysis of mature, mutant pollen indicated that it is deficient in long-chain lipids and has none of the lipoidic tryphine normally present on its surface. Immature mutant pollen grains have aberrant tryphine that disappears during pollen development. The sterile plants also lack stem waxes, and pollen from other wax-defective (eceriferum) mutants with reduced fertility has few of the lipid droplets normally present in tryphine. These results demonstrate that tryphine is critical for pollen-stigma interactions and suggest that tryphine lipids are required for fertilization, either by directly signaling the stigma or by stabilizing other tryphine components.
\end{abstract}

[Key Words: Pollen; Arabidopsis; compatibility; lipids; tryphine; eceriferum]

Received February 22, 1993; revised version accepted April 7, 1993.

Fertilization in flowering plants involves many cell-cell interactions, including adhesion of pollen grains to the -stigma surface, growth of pollen tubes through the pistil, migration of sperm cells within the pollen tube, and, ultimately, fusion of the sperm with egg cells; yet few of the molecules required for these interactions have been identified. As a first step in understanding these cellsignaling events, several sterile Arabidopsis mutants blocked in early steps of fertilization were identified. In this paper, one such mutant, defective in the interaction between pollen grains and stigma cells, is described.

The signaling events that occur during fertilization presumably require direct contact between cells and rely on molecules present in low abundance. Few of these molecules have been identified through in vitro reconstitution experiments, in part because manipulation of reproductive tissues is technically difficult. In contrast, genetic approaches depend solely on the function of molecules, rather than on their abundance, and allow cellcell communication events to be identified and characterized in living plants. Because most plant species are

\footnotetext{
${ }^{3}$ Corresponding author.
}

hermaphrodites (producing both sperm and eggs), mutant plants defective in either the male or the female reproductive process can be obtained and propagated readily.

Arabidopsis thaliana is well-suited to a genetic approach to fertilization. The advantages of its short life cycle, small physical size, and small genome size have been described (for review, see Meyerowitz 1989). Moreover, each flower is self-pollinated, and under laboratory conditions pollen from one plant rarely lands on another. Thus, mutant plants defective in any essential aspect of the fertilization process produce no seeds. Seed pod formation also can be used to monitor fertility; after fertilization, conspicuous seed pods are formed by the expansion of the pistil contained within each Arabidopsis flower. The penetrance of a mutation can be assessed easily because the plants flower continuously for several months, yielding hundreds of flowers. In fertile plants, these flowers each produce thousands of pollen grains and as many as 40 seeds. Sterile mutations occur at a high frequency in Arabidopsis. Nearly $1 \%$ of mutagenized seed pools yield plants that are completely sterile (see Materials and methods). Although many of these mutations disrupt formation of the male and/or female 
gametes, approximately one-tenth result in structurally normal flowers that are nonetheless functionally defective.

Adhesion of pollen grains to the stigma surface is mediated in part by an extracellular pollen coat, or tryphine (Dickinson and Lewis 1973a), and the pollen then absorbs water rapidly from the stigma, resulting in germination and tube growth (Heslop-Harrison 1979; Dickinson and Elleman 1985). In some species, pollen obtains water from a damp matrix that coats the stigma surface. In plants with dry stigmas, such as Arabidopsis, the tryphine from the pollen grain spreads along the proteinaceous coating of the stigma (Elleman et al. 1992), and water is transferred through this matrix from channels in the stigma cell wall (Roberts et al. 1984). Only pollen that forms these contacts becomes hydrated, whereas nearby pollen grains, not in physical contact with the stigma surface, remain desiccated (Sarker et al. 1988). Pollen germination is a highly selective process; pollen generally fails to germinate on the stigma of a distantly related species (for review, Zenkteler 1990), and germination of self-pollen is inhibited in many plants, a process known as self-incompatibility (for review, see Nasrallah et al. 1991). In Brassica species (close relatives of Arabidopsis), discrimination against foreign pollen or self-pollen is achieved at the stigma surface, either through control of pollen hydration or through control of subsequent pollen tube growth (Roberts et al. 1980; Ferrari et al. 1983; Sarker et al. 1988). Thus, through cellcell interactions that result in selective hydration, many plants promote germination of only compatible pollen grains on the stigma surface.

Not only is germination of inappropriate pollen blocked at the stigma surface, but in many species (including Brassica) stigma cells that contact incompatible pollen often synthesize callose, a $\beta-1 \rightarrow 3$ glucan that is ordinarily absent from the stigma cell wall (Dickinson and Lewis 1973b; Heslop-Harrison 1975). Extracts from incompatible pollen grains can recapitulate this response when applied to stigma cells (Heslop-Harrison et al. 1974; Kerhoas et al. 1983); however, the molecules that stimulate callose synthesis have not been identified. Callose is a normal component of the pollen tube wall but also appears in many tissues after pathogen invasion or wounding.

In this study a male-sterile Arabidopsis mutation that eliminates most of the tryphine from the pollen surface is described. The mutant pollen is viable but no longer interacts properly with Arabidopsis stigmas; pollen germination fails as a result of restriction of pollen hydration, and callose forms in stigma cells that contact the mutant pollen. This is the first example of a mutation that removes the pollen coat, and it provides an opportunity to characterize tryphine components and to discern their role in cell signaling during fertilization.

\section{Results}

Pollen from the popl mutant fails to germinate on the stigma surface

A collection of 175 infertile Arabidopsis plants was ob- tained by screening 25,000 mutagenized (M2) seeds (see Materials and methods). The pop1 mutant (defective in pollen-pistil interactions) is described here. This mutant was as healthy as wild-type plants but was completely infertile; no seeds were obtained from thousands of flowers. This mutation impairs only the male reproductive system. Both pop1 flower buds and mature pop1 flowers were fertilized successfully with wild-type pollen in $15 /$ 20 and $8 / 12$ crosses, respectively, resulting in $\sim 500$ seeds. In contrast, application of mutant pollen to either wild-type or pop 1 flower buds yielded no seeds $\mid 0 / 20$ and $0 / 13$ crosses, respectively). pop $1 /+$ heterozygotes are fertile and one-fourth of their progeny (320/ $1367=0.234 \mid$ have a sterile phenotype. Thus, the pop1 fertility defect results from a recessive mutation in a single genetic locus.

At the stigma surface (shown in Fig. 1A) germination of pollen results in the formation of a tube that serves to carry sperm cells to the ovules; one such tube, germinated in vitro, is shown in Figure 1C. These tubes contain high concentrations of callose, and this material can be visualized easily after staining with aniline blue and observing the resulting fluorescence (Fig. 1B; Eschrich and Currier 1964). Neither bright-field nor fluorescence microscopy detected pollen tubes on self-pollinated pop1 stigmas (Figure 1D,E). Similarly, application of pop1 pollen to wild-type pistils never resulted in visible pollen tubes. Thus, this early defect in pollen germination accounts for the observed male sterility in pop 1 plants.

The most striking phenotype associated with the pop1 defect was the appearance of callose on the stigma surface. Stigmatic papillae in direct contact with pop1 pollen (Fig. 1D,G), but not wild-type pollen (Fig. 1B,F) were highly fluorescent when stained with aniline blue, indicating that the mutant pollen stimulated an aberrant production of callose in stigma cells. The presence of callose was detectable within a few hours of pollination and persisted until the stigma degenerated 3-4 days later. This phenotype was observed in all sterile segregants from pop $1 /+$ heterozygotes and, thus, is attributable to the pop1 mutation. The presence of pop1 pollen on mutant or wild-type stigmas consistently stimulated callose production in stigma cells, but this was not observed in control crosses, in which wild-type pollen was applied to pop1 plants. Callose production did not occur when inviable wild-type pollen (treated with formaldehyde vapors) was applied to stigmas and, therefore, is not merely a consequence of a defect in pollen germination. Thus, pop1 plants make pollen that fails to germinate on the stigma and induces callose formation within stigma cells, both of which are characteristic of incompatible pollination.

\section{pop1 pollen germinates in vitro}

Pollen from most plant species germinates efficiently and forms pollen tubes when incubated in a simple medium consisting of sucrose, calcium, and borate (Brewbaker and Kwack 1963). Surprisingly, when pop1 pollen grains were incubated in this growth medium, germina- 
Preuss et al.
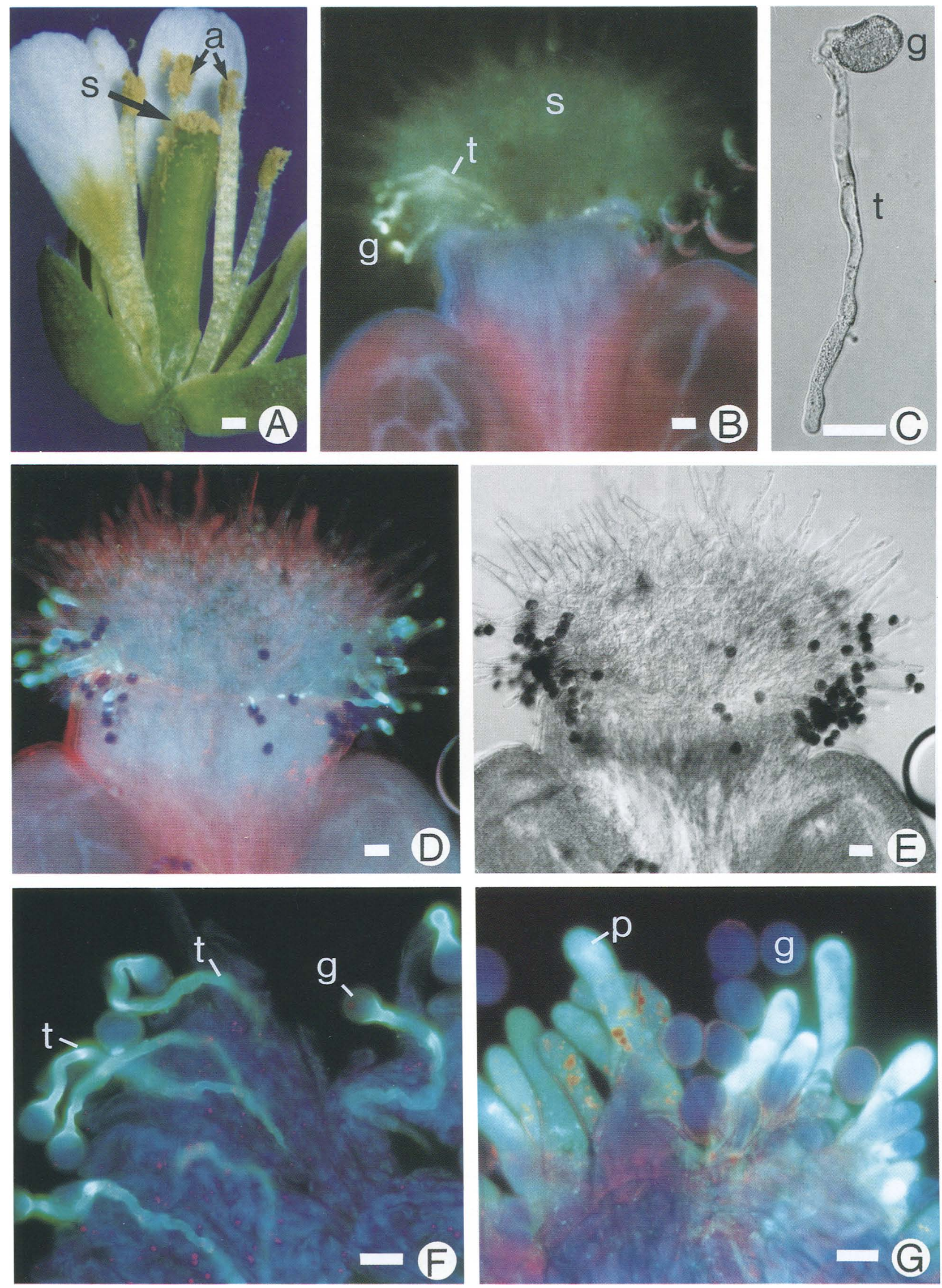

Figure 1. (See facing page for legend.) 


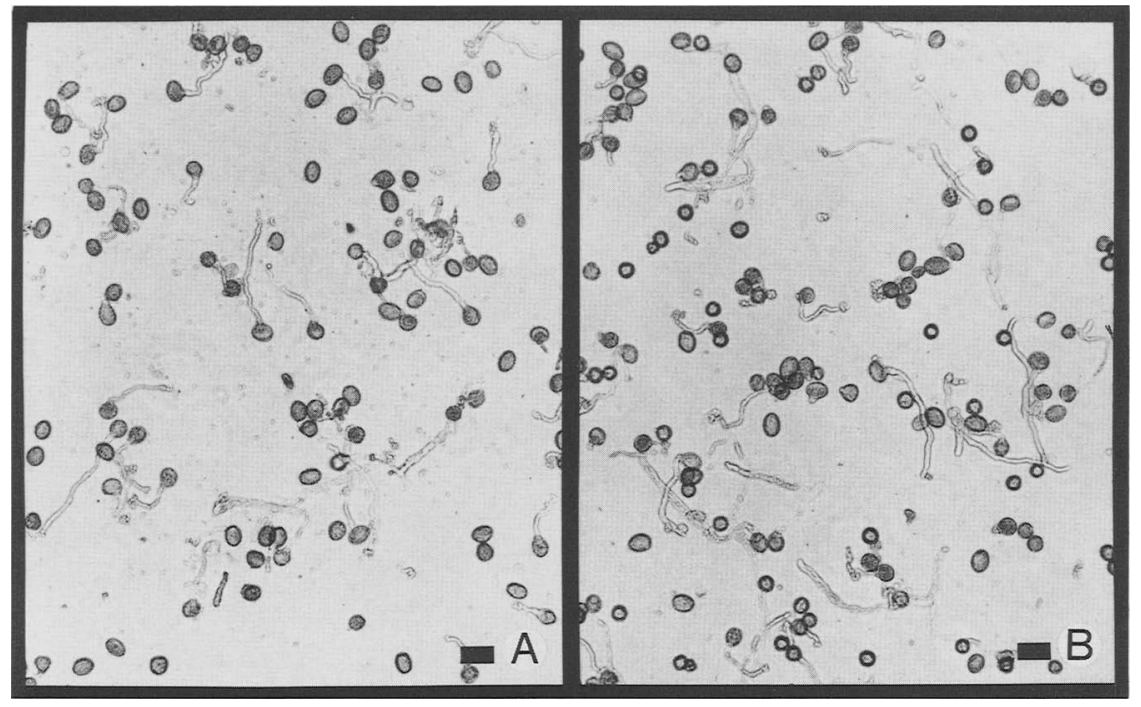

Figure 2. Germination of wild-type and pop1 pollen in vitro. Pollen tubes were observed after wild-type $(A)$ or pop1 $(B)$ pollen grains were incubated in pollen germination medium. Bars, $100 \mu \mathrm{m}$. tion of the mutant pollen was indistinguishable from wild type (Fig. 2). Germination of Arabidopsis pollen is relatively poor in this medium; nonetheless, after a $16-\mathrm{hr}$ incubation, $12 \%$ of wild-type pollen grains $(118 / 1019)$ and $18 \%$ of pop 1 pollen grains $(192 / 1064)$ germinated, and pollen tube lengths ranged from 0.1 to $0.5 \mathrm{~mm}$. These results indicate that pop1 pollen is viable and can synthesize, in vitro, all of the components necessary for pollen tube formation and growth.

popl pollen germination is rescued in vivo by humidity or by cofertilization with $\mathrm{POP}^{+}$pollen

Upon release from the anther, wild-type Arabidopsis pollen lands on the stigma surface and then begins to absorb water, becoming spherical rather than ovalshaped within a few minutes (a typical example is shown in Fig. 3A-E). A similar expansion of pop1 pollen was not detected (Fig. 3F-J), even when observations were extended to several hours after pollination. Of the hundreds of pollen grains observed, $>90 \%$ of the wild-type pollen was hydrated, whereas hydration of pop1 pollen was never detected.

This deficiency in pop1 pollen hydration on the stigma, coupled with the growth of the mutant pollen in vitro, suggested that the pop1 fertilization defect might be overcome by artificially hydrating the pollen grains. To test this possibility, pop 1 plants were shifted from standard growth conditions $(50-70 \%$ relative humidity) to a high-humidity chamber ( $90 \%$ relative humidity). Under these humid growth conditions, the mutant plants became fully fertile. In Figure 4, pop1 mutants are shown after growth in low humidity (Fig. 4A) and high humidity (Fig 4B); in the latter case, expanded seed pods indicate that fertility was restored. In addition, enclosing pop1 flowers in a polyethylene bag for as few as $5 \mathrm{hr}$ provided a sufficient increase in humidity to fully restore fertility. Sterility was also reversed when mature pop1 pollen, produced under relatively arid conditions, was transferred to humid conditions ( $18 / 26$ crosses), indicating that high humidity rescues pop1 pollen germination rather than its development. In every case, the mutant plants became sterile when returned to a low-humidity environment (see Fig. 4B). Thus, the pop1 mutation is conditional and completely reversible and represents the first conditional Arabidopsis mutation shown to affect pollen functions.

Although high humidity restores fertility to pop1 plants, interactions between the mutant pollen and the stigma surface are nonetheless aberrant. Under humid conditions callose still forms and persists on stigmatic papillae that contact pop1 pollen. In addition, whereas wild-type pollen tubes are intimately associated with stigma cells, growing between the cell wall and the plasma membrane of the papillae (Elleman et al. 1992), the pop1 pollen tubes produced in humid conditions grew more randomly, often appearing to be independent of the papillae cells. Growth of pop1 plants in a humid

Figure 1. Germination of wild-type and pop1 pollen on the stigma surface. $(A)$ Pollen is released from anthers onto the stigma surface. (C) Wild-type pollen tube after germination in aqueous growth medium. A fluorochrome in aniline blue preparations stains callose within pollen tubes; wild-type pollen produces tubes $(B, F$, turquoise staining), whereas no tubes are visible when pop1 plants are self-pollinated $(D, E)$. Within a few hours after pollination, callose forms on stigmatic papillae that contact pop1 pollen $(D, E, G)$ but not wild-type pollen $(B, F)$; stigmas shown in these panels were fixed 1 day after pollination. Staining techniques result in hydration of both the mutant and wild-type pollen grains, and other fluorochromes in the aniline blue preparation result in faint, dark blue staining of pollen grains. (a) Anther; $(\mathrm{g})$ pollen grain; (p) stigmatic papillae; $(\mathrm{s})$ stigma and $(\mathrm{t})$ pollen tube. Bars, $1 \mathrm{~mm}(A) ; 100 \mu \mathrm{m}(B$, $D, E) ; 50 \mu \mathrm{m}(C, F, G)$. 


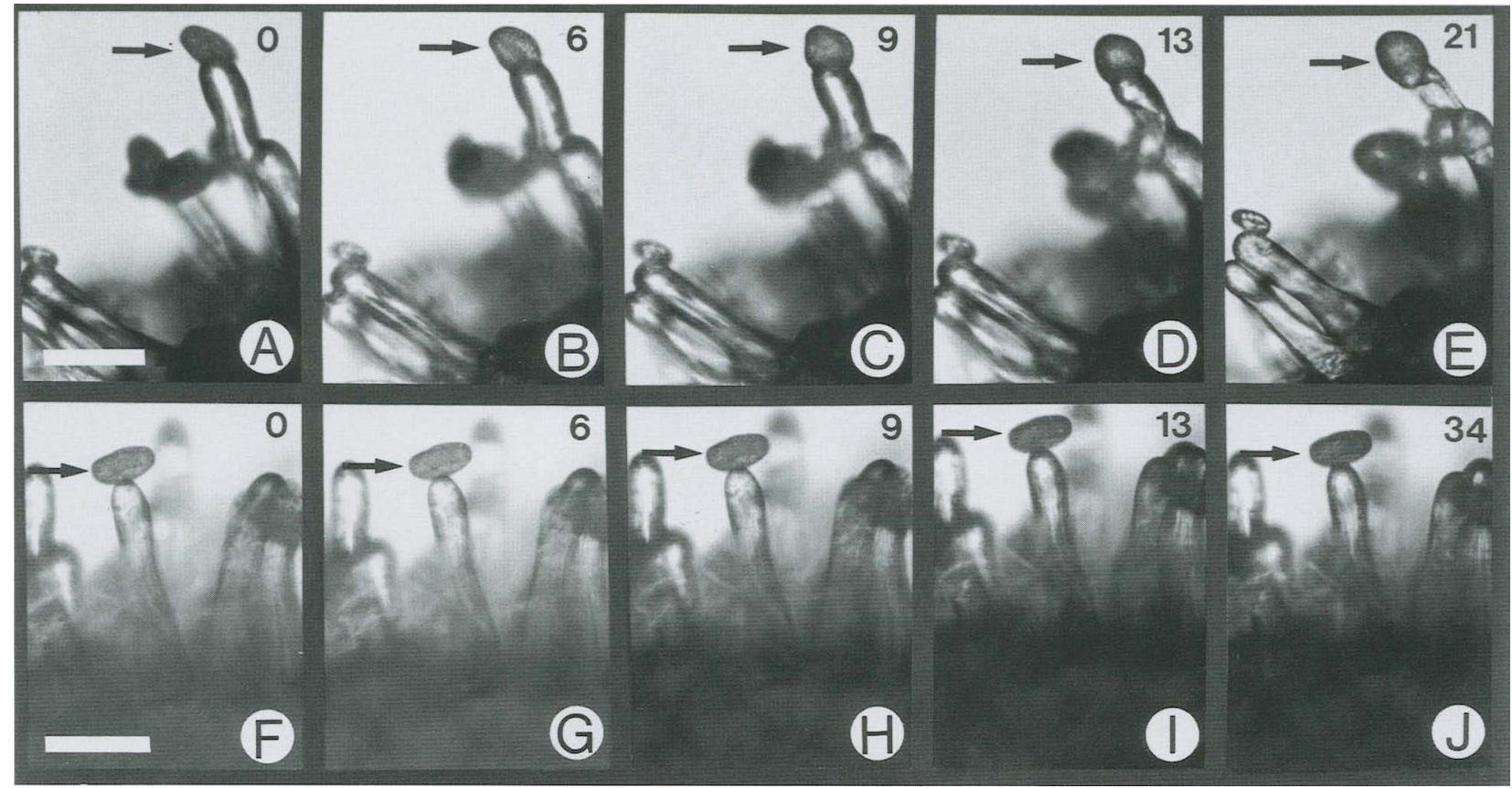

Figure 3. Hydration of wild-type, but not pop1, pollen occurs rapidly on the stigma surface. Wild-type pollen (arrows, $A-E$ ) expands within minutes when placed on a wild-type stigma surface. No change in pollen shape or size was observed in similar experiments with pop1 pollen $(F-I)$. Numbers (upper right) indicate time in minutes. The examples depicted here are representative of similar observations of $>100$ pollen grains. Bars, $100 \mu \mathrm{m}$.

environment therefore does not completely suppress all of the pop1 defects but, instead, allows hydration of the mutant pollen presumably through simple absorption of water vapor.

The experiments described above suggested that the stigma surface discriminates among pollen grains, resulting in hydration of wild-type but not pop1 pollen. In many species, hydration of foreign or incompatible pollen does not take place but can proceed if compatible mentor pollen is also applied to the stigma (Knox et al. 1987). Thus, to further characterize the requirements for hydration, pop1 flower buds were pollinated (at 50-70\% relative humidity/ with a mixture of wild-type and pop1 pollen, taking care to place the wild-type pollen near the
Figure 4. Humidity restores fertility in pop1 plants. pop1 plant grown in $50-70 \%$ relative humidity $(A)$ or in $90 \%$ relative humidity $(B)$. The arrow indicates the point at which the plant was returned to an arid environment. Expanded seed pods in $B$ indicate that fertilization has occurred.

mutant pollen grains. As expected, viable seeds were formed. Surprisingly, these seeds not only yielded fertile, pop1/ + plants, but also sterile, pop1/pop1 plants, indicating that the wild-type pollen promoted pop1 pollen germination. An equal mixture of the two types of pollen resulted in 4 homozygous mutant plants and 10 heterozygotes, suggesting that rescue of the mutant pollen was fairly efficient. Callose formation on the stigma was detected, but because pop 1 and wild-type pollen grains are indistinguishable in the light microscope, it was difficult to assess whether the callose on the stigma resulted from isolated clusters of pop1 pollen or from mixtures of mutant and wild-type pollen. These results indicate that interaction between $\mathrm{POP}^{+}$pollen and the stigma surface can result in hydration of nearby pollen grains, including defective grains that, by themselves, might fail to send appropriate signals. Mechanistically, rescue of the pop1 pollen might be achieved through diffusion of a wild-type pollen component. Alternatively, hydration of wild-type pollen in Arabidopsis might trigger hydration of nearby pollen grains, although this seems unlikely given the high specificity of pollen hydration in Brassica species. Other Arabidopsis mutants with defects in pollen hydration have been identified in another laboratory, and these mutants similarly can be rescued by wild-type mentor pollen (R.E. Pruitt, pers. comm.).

\section{pop1 mutants are deficient in wax production}

In addition to a defect in fertility, the original pop1 isolate also had a striking defect in the production of waxes on the surface of stems and seed pods. Epicuticular wax is composed of long-chain lipid molecules and is visualized easily on the surface of wild-type Arabidopsis stems as a dull, glaucous coating. In contrast, stems from the pop1 isolate were bright green and glossy in appearance (Fig. 5A), resembling wax-defective, or eceriferum (cer) mutants (Dellaert et al. 1979; Koornneef et al. 1989).
Scanning electron microscopy confirmed that the pop1 mutant (Fig. 5C) was completely devoid of the wax blooms present on wild-type stems (Fig. 5B; Koornneef et al. 1989). Crossing the pop1 mutant to a wild-type plant showed that this cer phenotype, like sterility, was completely recessive. Moreover, the sterile and cer phenotypes were tightly linked. Self-crossing of a pop $1 /+$ heterozygote yielded 1367 progeny, 320 of which were sterile and waxless, whereas the remainder were fertile and had a waxy cuticle. No recombination between the sterile and cer phenotypes was observed, indicating that these defects are tightly linked. Linkage of 0.073 map units $($ m.u. $)(<1 / 1367 \times 100 \%$ ) indicates that mutations could be within $\sim 15 \mathrm{~kb}$, assuming that recombination frequencies are uniform throughout the genome, with a genome size of $\sim 100,000 \mathrm{~kb}$ (Hwang et al. 1991) and genetic map of $\sim 500 \mathrm{~m}$.u. (Koornneef 1987), or $\sim 200$ $\mathrm{kb} / \mathrm{m} . \mathrm{u}$. These results strongly support a model in which defects in wax production and in fertility might be the result of the mutation of a single gene.

To explore further the relationship between fertility and wax synthesis, other cer mutants were characterized. Mutations in only 3 of the 21 cer genes (cer1, cer2, and cer6) completely eliminate wax structures on stems (Koornneef et al. 1989) and thus resemble the pop1 mutant. These mutants can be distinguished on the basis of their lipid content (Hannoufa et al. 1993). Chromatographic analysis of pop1 stem extracts indicated that like plants with defects in the cer2 or cer6 genes, pop $1 \mathrm{mu}-$ tants accumulate shorter lipids (24-27 carbons) but have low levels of the longest lipid chains (29-30 carbons; Table 1). In contrast, cer1 plants accumulate 30-carbon alcohols and aldehydes but are deficient in 29-carbon lipids (Hannoufa et al. 1993). Thus, the phenotype of the pop1 mutant, with respect to wax synthesis, closely resembles that of cer2 or cer6, suggesting that these gene products might affect the same biochemical step in lipid biogenesis.

In their screen for wax-defective plants, Koornneef et al. (1989) discarded mutants with severe fertility defects;
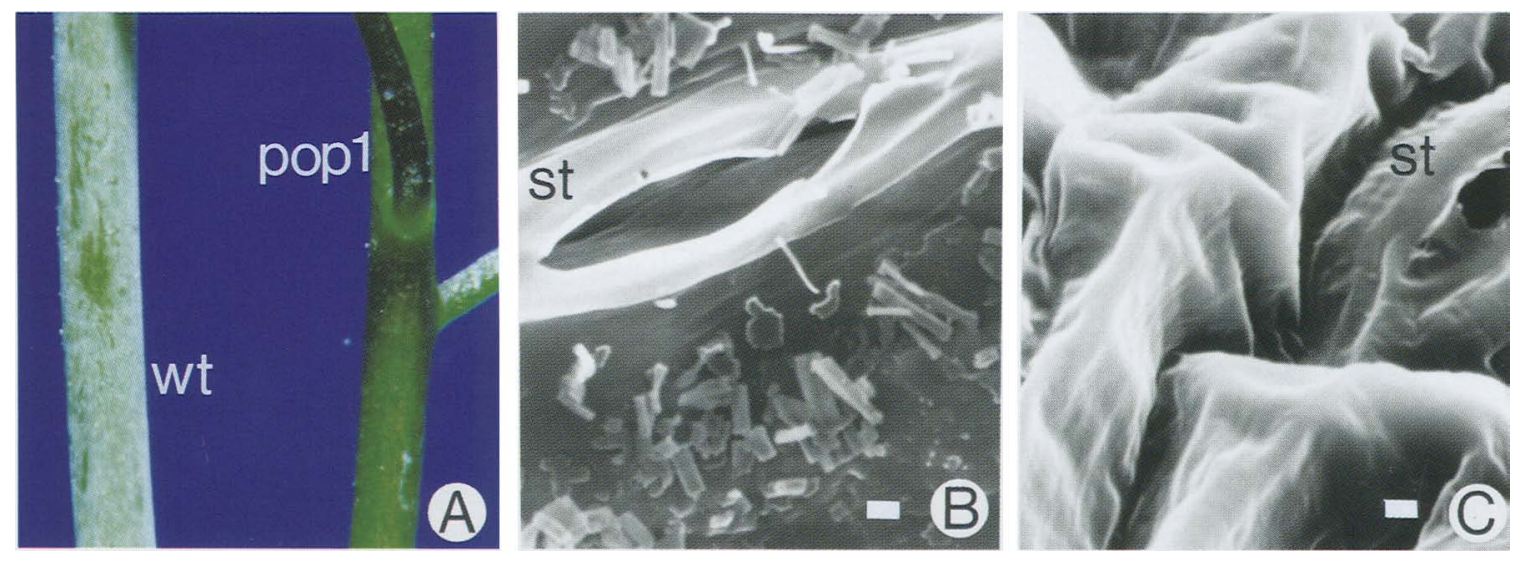

Figure 5. pop1 mutants are defective in epicuticular wax production. pop1 plants are deficient in the waxes that coat wild-type stems $(A)$, and scanning electron microscopy confirms that plates of wax are present on wild-type stems $(B)$ but are absent from pop1 stems $(C)$. As a size reference, two stomates (st) were included in the photographs. Bars, $1 \mu \mathrm{m}$. 
Table 1. Epicuticular wax components from wild-type, popl, and cer stems

\begin{tabular}{|c|c|c|c|c|c|}
\hline $\begin{array}{l}\text { Number of } \\
\text { carbon atoms }\end{array}$ & Wild type & pop1 & cer1 & cer2 & cer6 \\
\hline 24 & 0.6 & $22.5(38)$ & $1.5(2.5)$ & $7.3(12)$ & $7.5(12)$ \\
\hline 26 & 4.6 & $61.5(13)$ & 9.0 (2) & $57.1(12)$ & $40.5 \quad(9)$ \\
\hline 27 & 0.3 & $2.8 \quad(9)$ & $0 \quad(-1$ & $0 \quad 1-1$ & 3.9 (13) \\
\hline 28 & 11.5 & $5.1|0.5|$ & $8.6(0.8)$ & $26.5 \quad 2\}$ & 29.1 (2) \\
\hline 29 & 64.3 & $1.7(0.03)$ & $12.0(0.2)$ & $1.0(0.02)$ & $17.2\{0.25\}$ \\
\hline 30 & 17.5 & $3.5(0.2)$ & $49.7 \quad(3)$ & $1.0(0.06)$ & $<0.2(<0.01)$ \\
\hline
\end{tabular}

The sum of alcohol, aldehyde, alkane, carboxylic acid, and ketone quantities is reported. Values represent percent of total lipid present in chloroform extracts from stems. Shorter chain lipids (14-22 carbons) and 25-carbon chains accounted for $<2 \%$ of the total material and are not shown. Parentheses indicate fold-change from wild-type extracts.

nonetheless, most of the cer6 (but not cer2) mutants that they recovered had reduced seed yields when grown in greenhouses. In contrast, in the low humidity chamber used here, both the cer2 and cer6 mutants were completely sterile. As with pop1, sterility was limited to the male reproductive system. Mutant pollen failed to fertilize wild-type flower buds (12 crosses each yielded no seeds|, whereas wild-type pollen efficiently fertilized the mutants $(8 / 11$ cer 2 and $8 / 12$ cer 6 crosses were successful). The reduced seed yields observed in cer mutants was previously attributed to excessive loss of water from the mutant plants (Dellaert et al. 1979; Koornneef et al. 1989); however, the crosses described above argue strongly that these mutants are capable of producing seeds if provided with the proper pollen.

Crosses between the cer2 mutant and pop1 in high humidity/ yielded progeny that produced wax and were fully fertile. Similar crosses to the cer 6 mutant resulted in sterile progeny with a cer phenotype. In addition, the cer6 and pop 1 defects are tightly linked; $23 / 23$ self-progeny from a cer6/pop1 heterozygote had a cer phenotype. Thus, pop1 represents a new allele of the CER6 gene; henceforth called cer6-2. Further examination of the cer mutants indicated that the pollen from cer2 and cer6-1 plants also failed to hydrate and thus germinate on the stigma surface but grew well in liquid medium. Consistent with previous observations (Koornneef et al. 1989), placing these cer mutants in a humid environment did not reverse the wax defect but completely restored fertility. The cer2 and cer6-1 mutants differed from cer6-2 (pop1) plants in one respect; callose production within the stigmatic papillae was less dramatic. Thus, although pop1 and cer6-1 are allelic, the pop 1 mutation likely represents a more extreme allele.

\section{Long-chain lipids are deficient in popl pollen grains}

The striking similarities between the cer2, cer6-1, and pop1 (cer6-2) mutants, including their defects in wax synthesis and pollen germination, suggested that lipid biogenesis might play a role in pollen-stigma communication. The lipid content of wild-type Arabidopsis pollen had not been characterized previously, and to test whether long-chain lipids were present, pollen lysates were prepared and extracted with chloroform, and the components in the organic phase were subsequently separated by gas chromatography. The chromatogram shown in Figure 6 indicated that wild-type pollen contains several long-chain lipid compounds. The identity of these molecules was confirmed by analysis of their of mass spectra, and their relative abundance is shown in Table 2 .

To test whether the lipid content of pop1 pollen might be aberrant, lysates were prepared in parallel and were compared to extracts from wild-type pollen. Although

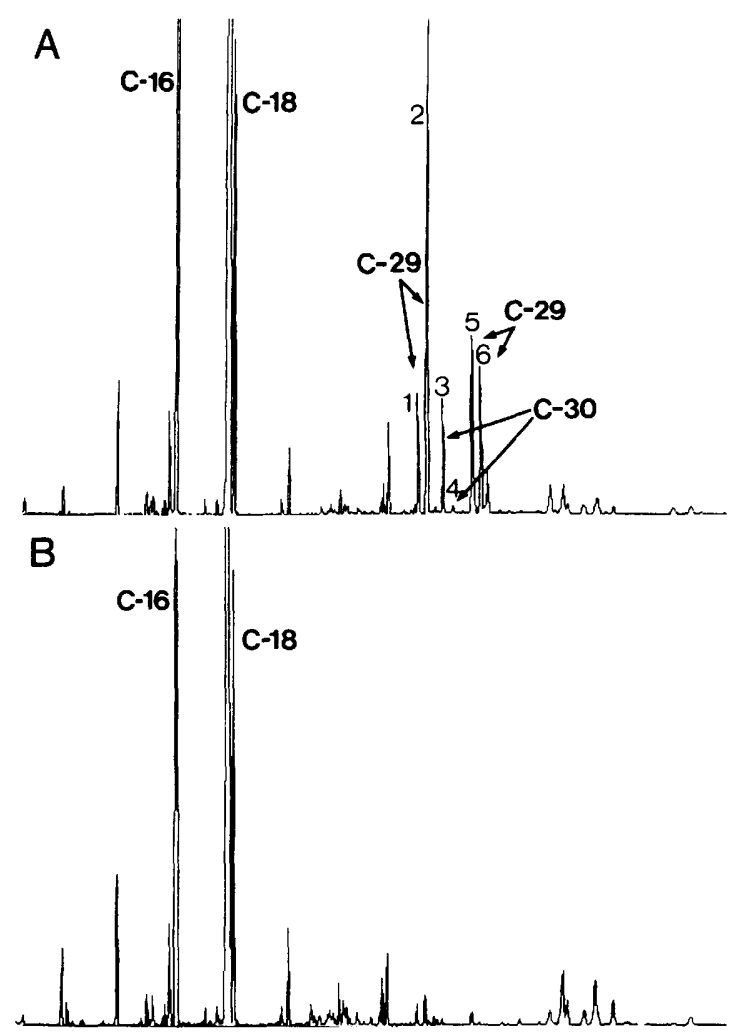

Figure 6. pop1 pollen is deficient in long-chain lipids. Pollen lysates were extracted with chloroform and analyzed by gas chromatography followed by mass spectroscopy. The wild-type extract $(A)$ contains 29 - and 30-carbon lipids (identified in Table 2), whereas these compounds are virtually absent from the pop1 extract $(B)$. 
Table 2. Lipid content in extracts from popl and wild-type pollen

\begin{tabular}{lllccl}
\hline Peak number & $\begin{array}{l}\text { Number of } \\
\text { carbon atoms }\end{array}$ & Compound & Wild type & pop1 & \\
\hline & 16 & hexadecanoic acid & 19 & 19 & $(1)$ \\
1 & 18 & octadecanoic acid & 62 & 67 & $(1.1)$ \\
2 & 29 & nonacosene & 0.86 & 0.15 & $(0.17)$ \\
3 & 29 & n-nonacosane & 4.45 & 0.34 & $(0.08)$ \\
4 & 30 & triacontene & 0.92 & 0.05 & $(0.05)$ \\
5 & 30 & n-triacontane & 0.07 & 0.0 & $(-)$ \\
6 & 29 & 14- and 15-nonacosanol & 2.04 & 0.19 & $(0.09)$ \\
\hline
\end{tabular}

Peak numbers refer to those indicated in Fig. 6. Values represent percent of total lipids in pollen lysates. Identification of compounds was determined by analysis of their mass spectra, and quantitation is based on integration of total peak area from the ion chromatogram. Parentheses indicate fold-change from wild-type extracts.

the mutant pollen grains had normal levels of shortchain lipids (16 and 18 carbons), long-chain molecules were low in abundance (Fig. 6). Twenty-nine-carbon molecules ( $n$-nonacosane, nonacosene, 14- and 15-nonacosanol, and 15-nonacosanone) were easily detected in the wild-type sample, but only a small fraction of these lipids $(2-17 \%$ of wild-type levels) was found in the pop1 extract, and 30-carbon molecules were present at $<5 \%$ of the wild-type levels (Table 2). These results are completely consistent with the analysis of epicuticular waxes, indicating that pop1 mutants extend few lipid chains to a length of 29-carbon atoms.

Pollen grains have an inner cell wall composed of pectin and cellulose (intine), an external wall formed by a complex polymer known as sporopollenin (exine), and a layer of tryphine, composed of lipid and protein molecules, embedded in the exine surface (for review, see Dobson 1989). The function of tryphine (also known as pollenkitt) is not fully understood; it may protect pollen from damage, attract insect pollinators, or promote adhesion of pollen to the stigma surface. Moreover, tryphine may play a direct role in cell signaling, because material extracted from the pollen surface stimulates callose production when applied to plants (Heslop-Harrison et al. 1974; Kerhoas et al. 1983).

To discern whether tryphine structure was altered in cer2, cer6-1, or pop1 (cer6-2) mutants, flowers from each of the mutant plants were fixed and prepared for electron microscopy, using osmium tetroxide, a lipophilic stain. This analysis indicated a dramatic defect in pop1 pollen grains. As shown in Figure 7, tryphine was apparent on the surface of wild-type pollen grains (Fig. 7A) but was virtually absent from the pop1 pollen (Fig. 7B). Exine structure appeared normal both in thin sections (Fig. 7B) and in scanning electron micrographs (Fig. 7C-D). Pollen from cer2 and cer6-1 mutants showed a more subtle defect. Although tryphine was present, the number and size of the lipid droplets within the tryphine were significantly reduced (Fig. 7E-F), providing further evidence that the cer6-1 allele is less extreme than pop1 (cer6-2). Each of these cer mutants has defects in long-chain lipid biosynthesis, and the observation that tryphine structure is also aberrant strongly implicates long-chain lipids in the formation of structurally and functionally normal pollen.

In wild-type pollen grains, tryphine is deposited soon after meiosis by diploid tissue in the anther (tapetum) and becomes compact during anther dehydration (Dickinson and Lewis 1973a). Surprisingly, tryphine was observed on the surface of pop1 pollen grains early in their development, although this material apparently lacked lipid droplets (Fig. 7G-H). As the mutant pollen developed, progressively less tryphine was detected until none remained at maturity. These results suggest the pop1 mutation alters the stability of tryphine or its ability to remain bound to the pollen surface.

\section{Discussion}

The pop1 (cer6-2) mutation results in pollen that behaves, in every respect, as if it no longer sends appropriate signals to the Arabidopsis stigma surface. At low humidity, hydration, and consequently germination, of the mutant pollen does not occur on the stigma. Moreover, the presence of callose within stigmatic papillae indicates that interactions with pop1 pollen are aberrant. These results argue strongly that this mutation disrupts pollen-stigma communication.

In vitro germination substantiates that pop1 pollen is viable. Moreover, the in vivo fertility defect is reversed when pop1 pollen is mixed with wild-type pollen grains or when the mutant plants are shifted to a humid environment. These results indicate that, once hydrated, growth of pop1 pollen is normal. Unlike the previously isolated temperature-sensitive defects in Arabidopsis flower development (Bowman et al. 1991), pop1 represents a conditional, male-sterile mutation that specifically influences pollen function.

A deficiency in wax on the stems of pop1 plants provided the first indication that long-chain lipid biosynthesis might be required for pollen function. Five wax-defective, cer mutations were shown previously to affect seed yields, but this defect was attributed to increased transpiration from the mutant plants (Dellaert et al. 1979; Koornneef et al. 1989). Under the growth conditions used here, two of these cer mutants (cer2 and cer6- 
Figure 7. Ultrastructural analysis of the pollen surface. Transmission electron microscopy was used to visualize wall structure in wild-type $(A)$, pop $1(B), \operatorname{cer} 2(E)$, and cer6 $(F)$ pollens. Osmium tetroxide was used to stain lipids, and proteins were stained with uranyl acetate and lead citrate. Tryphine (tr) is notably absent from the surface of pop1 pollen $(B)$, whereas the size and abundance of tryphine lipid droplets (arrows in A) are reduced in cer2 and cer6 mutants. Exine structure, visualized by scanning electron microscopy, was identical in wild-type $(C)$ and pop1 pollen $(D)$. Early in pop1 pollen development, some aberrant tryphine (arrowheads) is present $(G)$, which begins to disappear as the pollen matures $(H)$ and the tapetum (tp) degenerates. (e) External cell wall (exine); (i) internal cell wall (intine); (c) cytoplasm; $($ tr) tryphine; $($ tp $)$ tapetum. Bars, 0.5 $\mu \mathrm{m}(A, B, E, F) ; 2 \mu \mathrm{m}(C, D)$; and $1 \mu \mathrm{m}(G, H)$.

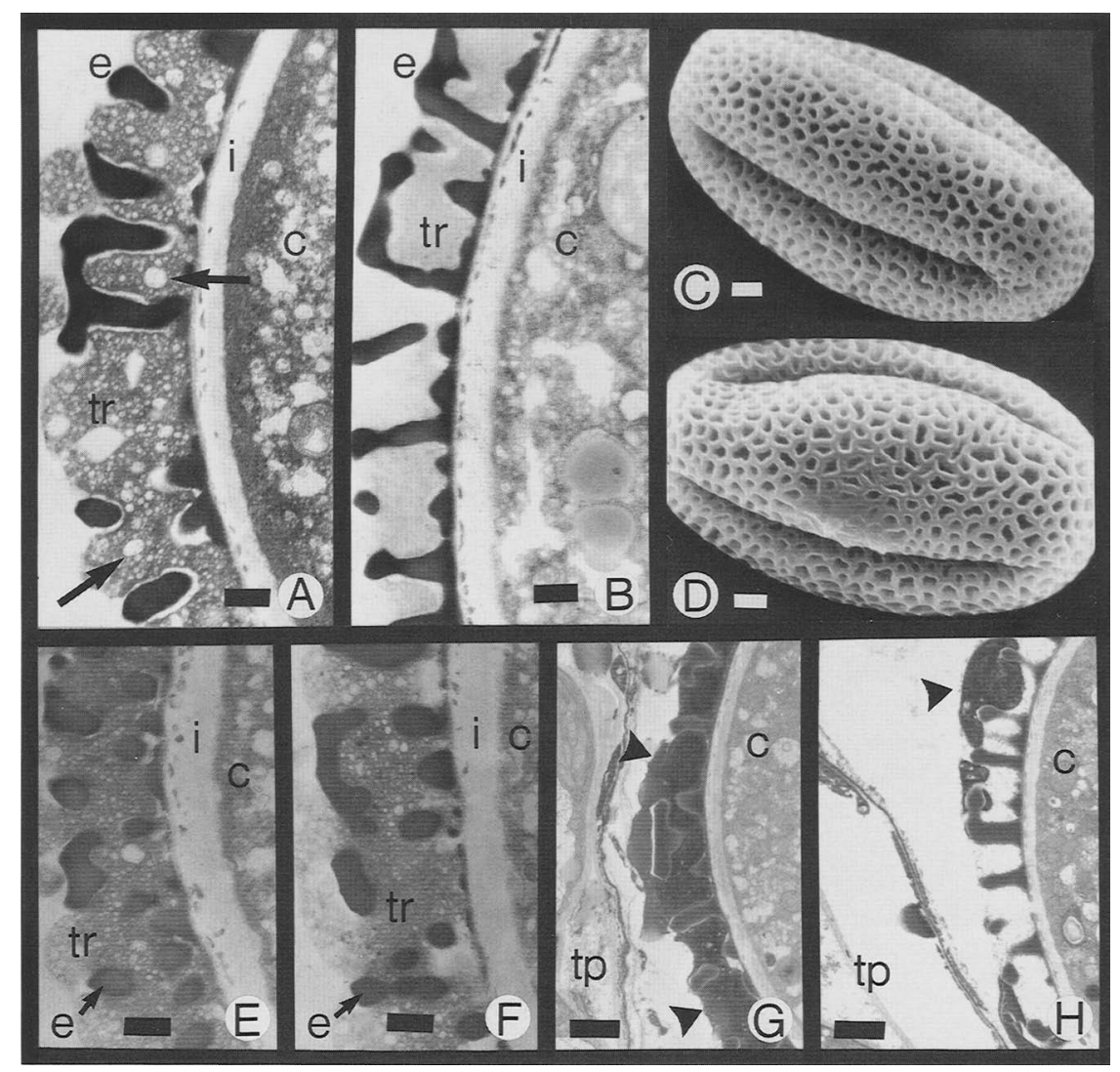

1) were shown to be male-sterile, producing pollen that has pop1-like defects and fails to germinate on the stigma surface. The phenotype of the pop1 mutant differed from these cer mutants in one respect; only pop1 pollen stimulated a dramatic production of callose within stigma cells. Complementation tests and linkage analysis indicated that pop1 is a more deleterious allele of the CER6 gene (cer6-2); CER6 was mapped to chromosome 1 previously (Koornneef et al. 1989). Thus, alterations in either the CER2 or CER 6 genes impair both wax synthesis and fertility, strongly indicating a role for long-chain lipids in the development of functional pollen.

Wild-type, but not pop1, pollen was found to contain significant quantities of long-chain lipid molecules. As in maize (Bianchi et al. 1990), these lipids are predominantly alkanes, secondary alcohols, and ketones, rather than the aldehydes and primary alcohols found on the surface of stems (Hannoufa et al. 1993). Most likely, these long-chain pollen lipids reside within the tryphine that coats the surface of pollen grains. No tryphine remains on the surface of pop1 pollen grains at maturity; less severe tryphine alterations were also detected in cer2 and cer6-1 pollen. These observations thus provide direct genetic evidence that, at low humidity, tryphine is required for pollen germination on the stigma and indicate that pollen-stigma signaling is mediated, at least in part, through tryphine components. After pollen con- tacts the stigma, some of the tryphine spreads along the proteinaceous coating of the stigma surface, establishing a region of continuity (Elleman et al. 1992). Long-chain lipids might be required to mobilize tryphine components, to signal stigma cells directly, or to provide a matrix for assembly of other essential tryphine molecules.

The results described above suggest a general model for pollen hydration in Arabidopsis (Fig. 8). First, compatible wild-type pollen must signal the stigma surface, resulting in the transfer of water through channels in the stigma cell wall (Fig. 8A). In cer2, cer6-1, and pop1 (cer62) mutants, alterations in tryphine structure disrupt this communication, consequently eliminating pollen hydration. It is conceivable that the loss of tryphine might affect hydration indirectly, resulting in highly desiccated pollen grains that require more than normal amounts of water to become fully hydrated. The failure to observe any decrease in the diameter of the mutant pollen relative to wild type, or, at low humidity, to detect even partial hydration of the mutant pollen by stigma cells (Fig. 3) argues against this interpretation. More likely, tryphine is required directly to establish communication between pollen and the stigma surface.

The tryphine defect in pop1 plants is clearly more extreme than that observed in cer2 and cer6-1 mutants. Presumably, the surface of pop1 pollen is so aberrant that it strongly induces callose production on the stigma, a response that was less dramatic after pollination with 
A
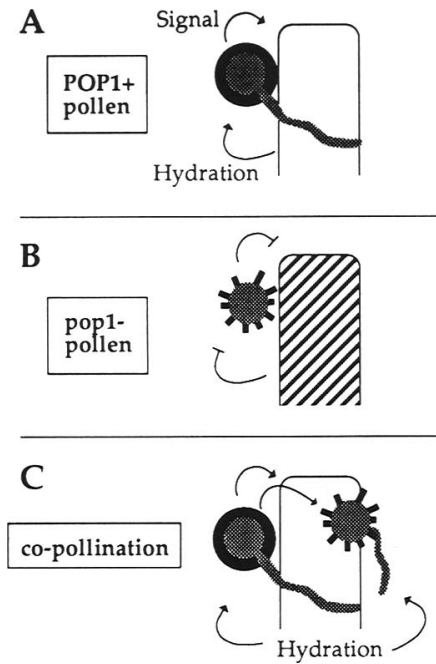

Figure 8. Interaction of pollen with the stigma surface. Wildtype pollen $|A|$ signals the stigma surface, resulting in hydration. Defective signaling occurs with cer2, cer6-1, or pop1 (cer62) pollens (as a result of tryphine defects), resulting in no pollen hydration $(B)$. pop1 pollen also stimulates callose production within stigma cells (shading). Copollination with wild-type and pop1 pollen results in germination of both pollen grains, suggesting that the pollen signal diffuses $(C)$.

cer2 or cer6-1 pollen (Fig. 8B). Although the presence of callose in stigma cells is correlated with incompatible pollination (especially in Brassica), it is not produced in every case, and its role therefore remains unclear. Callose may serve as a general defense against pathogens, and only those pollen grains that differ significantly from compatible pollen might stimulate callose production in the stigma. High humidity rescues pop1 sterility and presumably bypasses potential callose barriers by allowing pollen tube growth that is independent of a close association with the papillae cells.

Finally, copollination experiments suggest that the pollen signal can diffuse and, thus, promote the growth of nearby pollen grains (Fig. 8C). This could be accomplished through spreading of wild-type tryphine to the mutant pollen grains. Although molecules that mediate the pollen signal have not been identified, recent experiments indicate that low levels of flavonoids are required for pollen germination in maize, petunia, and tobacco, suggesting a role for these compounds in pollen-pistil signaling (Mo et al. 1992; Taylor and Jorgensen 1992; Ylstra et al. 1992). pop1 and other mutants with similar phenotypes provide an opportunity for testing the effects of such molecules, or other plant factors in vivo; any substance that signals the stigma and, hence, stimulates pollen hydration should promote pollen germination and thus seed formation.

The germination of pop1 pollen in humid conditions not only supports the idea that the mutant pollen, if hydrated, is fully functional, but also allows propagation of pop1 plants. This conditional, male-sterile mutant should greatly facilitate construction of Arabidopsis strains. Fertilization of the sterile plants with $P O P 1^{+}$ pollen will result in both heterozygous hybrids and pop1 homozygotes; heterozygotes can be identified subsequently on the basis of epicuticular wax production, a phenotype that is apparent at an early stage. In crop plants, much effort has focused on identifying male-sterile mutations that allow breeders to control hybrid production. In addition, Mariani et al. (1990) genetically engineered male sterility by expressing a cytotoxic ribonuclease gene within the tapetum, completely eliminating pollen production. Propagation of these natural and engineered male-sterile mutants requires either manual pollination /which is tedious and expensive on a large scale) or restoration of fertility, either through a compensating mutation or expression of a ribonuclease inhibitor (Mariani et al. 1992). By using the same tissuespecific promoter, expression of antisense CER2 or CER6 messages, solely within the tapetum, should be possible. This alteration presumably would result in conditional male sterility without eliminating the waxy coating on stems and would thus greatly facilitate the propagation of any plant that requires these genes, or their homologs, for pollen function.

The similarities between the pop1 mutation and the self-incompatibility (SI) studied in many Brassica species is striking. Both regulate pollen germination at the stigma surface, often through control of pollen hydration. In addition, there are many examples of callose production in stigma cells that directly contact incompatible, self-pollen. High humidity results in efficient germination of pop1 pollen and can sometimes rescue selfincompatible pollen, although in the latter case, pollen tube growth is eventually arrested. Finally, copollination with compatible mentor pollen can overcome SI (Knox et al. 1987; Sarla 1988), resembling the effect of wild-type pollen on pop1 pollen germination. SI responses differ from pop1 pollinations in one respect: Immature flower buds from self-incompatible plants often can be self-pollinated, but neither mature nor immature Arabidopsis stigmas promote germination of pop1 pollen. Nonetheless, SI could serve as a model for many aspects of pop1 pollen rejection in Arabidopsis. Recently, a multigene family involved in pollen-stigma interactions in Brassica was identified (for review, Nasrallah et al. 1991). These highly polymorphic genes encode glycoproteins that are present at high levels in the stigmatic papillae. Genes similar in sequence are present in Arabidopsis, and at least one of these, AtS1, is expressed in flower buds (Dwyer et al. 1992). The AtS1 gene product thus potentially functions at the stigma surface and might regulate interaction between pollen and the stigmatic papillae.

Clearly, the CER2 and CER6 genes are required both for stem wax formation and for pollen function. At the biochemical level, these gene products presumably mediate the addition of 2-carbon acetyl groups to long-chain fatty acids. In Arabidopsis seeds, fatty acid elongation is regulated by several genes (Lemieux et al. 1990), and in leaf tissue, long-chain fatty acids apparently are synthesized by an enzyme complex (Bessoule et al. 1989). The 
phenotypic differences between the cer6-1 and pop1 (cer6-2) mutant strains indicate that the pop1 allele is more severe, perhaps representing a null mutation. In addition to CER2 and CER6 mutations, alterations in three other genes required for wax synthesis, CER3, CER8, and CER10, result in low seed yields (Dellaert et al. 1979; Koornneef et al. 1989), although none of these alleles are known to be null or to specifically affect pollen function. The common feature of all of the partially sterile cer mutants is a reduction in 29-carbon alkane, alcohol, or ketone levels (Hannoufa et al. 1993), indicating that these long-chain lipid molecules are necessary for pollen-stigma interactions, either for directly signaling the stigma or for stabilizing other essential molecules. Further characterization of the CER gene products, including identification of their biochemical functions, should elucidate the role of lipids in pollen-stigma communication, not only in Arabidopsis, but in other angiosperms as well.

\section{Materials and methods}

\section{Genetic techniques}

Strains were Landsberg erecta; cer mutants were obtained from the Arabidopsis Stock Center (Columbus, $\mathrm{OH}$ ). For crosses, anthers were removed from flowers before pollen maturation (dehiscence), and immature stigmas were dusted with pollen immediately or 2-3 days later (mature). All pop1 analyses were on segregants from a strain twice backcrossed to wild-type. Growth conditions were 24-hr fluorescent lighting 85 microEinsteins per square meter $\left.\left(\mu \mathrm{E} / \mathrm{m}^{2}\right)\right]$ at $18-22^{\circ} \mathrm{C}$ and $50-70 \%$ relative humidity (ambient conditions) or $90 \%$ relative humidity (Conviron chamber). Mutants were obtained by immersing seeds in $4 \mathrm{~mm}$ ethyl nitrosourea for $24 \mathrm{hr}$ at room temperature. Mutagenized (M1) seeds $(60,000)$ were grown, and plants generated from the resulting self-seeds (M2) were screened for sterile mutations at a density of $\sim 500$ seeds $/ \mathrm{ft}^{2}$. Reciprocal outcrosses indicated $\sim 40 \%$ of the mutants were male-sterile (most of which produced no pollen) and $10 \%$ were female-sterile. Homozygous pop1 seeds are strain WNN6.

\section{Microscopy}

Callose staining (Eschrich and Currier 1964) was performed on flowers fixed for $1 \mathrm{hr}$ in chloroform/ethanol/acetic acid [/vol/ vol) $6: 3: 1$, incubated for $10 \mathrm{~min}$ in $4 \mathrm{M} \mathrm{NaOH}$, then neutralized in $50 \mathrm{mM}$ phosphate buffer $(\mathrm{pH} 7.5)$, and stained with aniline blue at $50 \mu \mathrm{g} / \mathrm{ml}$ in $50 \mathrm{mM}$ phosphate buffer $(\mathrm{pH} 7.5)$. Fluorescence was observed on a Zeiss Axiophot microscope with a Zeiss $365 \mathrm{G}$ filter. Pollen hydration was assessed by light microscopy (Zeiss Axiophot); before hydration, Arabidopsis pollen has three conspicuous furrows and is oval-shaped. Hydration of wild-type pollen occurs within minutes of pollination, resulting in a twofold increase in pollen diameter, disappearance of the furrows, and formation of spherical pollen grains. For scanning electron microscopy, fresh stems and flowers were collected, placed on stubs coated with double-stick tape, and gold-coated to $8 \mathrm{~nm}$ with a Polaron E5400 sputter coater. Observations were with a Philips SEM505 microscope. For transmission electron microscopy, mature anthers and developing flower buds were fixed in $3 \%$ glutaraldehyde (EM Sciences, Ft. Washington, PA), $100 \mathrm{~mm}$ phosphate buffer (pH 7) for $5 \mathrm{hr}$ at $20^{\circ} \mathrm{C}$, then overnight at $4^{\circ} \mathrm{C}$. Subsequently, tissues were fixed in $2 \%$ osmium tetroxide (EM Sciences), $100 \mathrm{~mm}$ phosphate buffer $(\mathrm{pH} 7)$ for $2 \mathrm{hr}$ at $20^{\circ} \mathrm{C}$, washed in several changes of boiled, distilled water, and then incubated in $1 \%$ uranyl acetate for $30 \mathrm{~min}$. Dehydration was in a graded ethanol series 130 $100 \%$, and embedding was performed as described previously (Oliveira et al. 1983), except $n$-octenyl succinic anhydride was substituted for hexenyl succinic anhydride. The resin was polymerized in aluminum weighing dishes for 2 days at $60^{\circ} \mathrm{C}$. Thin sections measuring $60-90 \mathrm{~nm}$ (silver-gold interference color) were prepared with a Reichert-Jung Ultracut E microtome, and observations were on a Philips TEM410 microscope.

\section{Pollen preparation and extraction of lipids and waxes} from pollen and stems

Pollen was removed from mature flowers by suspending them in pollen germination medium $\left(17 \%\right.$ sucrose, $2 \mathrm{mM} \mathrm{CaCl}_{2}, 1.65$ $\mathrm{mM}_{3} \mathrm{BO}_{3}$ at $\mathrm{pH}$ 7), mixing vigorously, and then withdrawing the pollen suspension with a pipette. Purified pollen was then collected by centrifugation in a microcentrifuge $(5000 \mathrm{~g})$. For germination assays pollen was suspended in germination medium at 100 grains $/ \mu \mathrm{l}$ and incubated in microtiter wells for $12 \mathrm{hr}$ at $20^{\circ} \mathrm{C}$. Pollen lipid analysis (Fig. 6) was performed on $5 \mathrm{mg}$ of purified pollen (from 1500 flowers), and unambiguous identification of long-chain lipid compounds (Table 2) required $15 \mathrm{mg}$ of purified pollen. Pollen pellets were suspended in $40 \mu \mathrm{l}$ of ice-cold lysis buffer $(50 \mathrm{~mm}$ Tris- $\mathrm{HCl}, 5 \%$ glycerol, $100 \mathrm{~mm} \mathrm{KCl}$, $10 \mathrm{mM}$ EGTA at $\mathrm{pH} 8.0)$ containing protease inhibitors $(5 \mu \mathrm{g} / \mathrm{ml}$ of antipain, leupeptin, and pepstatin, $5 \mathrm{~mm} \epsilon$-amino- $n$-caproic acid, $1 \mathrm{~mm}$ phenylmethylsulfonyl fluoride, $1 \mathrm{mM}$ benzamide, and $10 \mu \mathrm{M} p$-aminobenzamidine). The suspension was Dounce homogenized (on ice) vigorously for $4 \mathrm{~min}$ in a microcentrifuge tube, and the cell lysate was suspended in $1 \mathrm{ml}$ of lysis buffer with protease inhibitors and transferred to a $15-\mathrm{ml}$ Corex tube containing $2 \mathrm{ml}$ of chloroform and $2 \mathrm{ml}$ of methanol. The extract was mixed, and the phases were separated in a tabletop clinical centrifuge. The chloroform phase was subsequently extracted once with water and was then collected and evaporated under nitrogen. Chromatographic analysis was performed as described previously (Hannoufa et al. 1993) by silylation with bis(trimethylsilyl)-trifluoroacetamide (BSTFA). The presence of long-chain fatty alcohols was detected as fatty acids after treatment with $1 \mathrm{~N} \mathrm{HCl}$ in methanol (Hannoufa et al. 1993); these long-chain fatty alcohols were present in trace amounts. The quantitations were based on an internal standard of nonadecane. Analysis of epicuticular wax was performed similarly, except wax was collected by immersing 1 gram of stems into chloroform for $20 \mathrm{sec}$.

\section{Acknowledgments}

We are grateful to M. Campbell, A. Lloyd, S. Long, L. Miller, M. Schena, and V. Walbot and anonymous referees for careful reading of this manuscript; to A. Lloyd, J. Mulligan, and M. Thomas, who generously provided mutagenized Arabidopsis seeds; and to F. Thomas for assistance with electron microscopy. We also thank D. Meinke and B. Pruitt for helpful advice. D.P. is a National Science Foundation (NSF) Postdoctoral Fellow, and this work was supported by NSF grant DMB-9106011 to R.W.D. and by a Natural Sciences and Engineering Research Council of Canada grant to B.L.

The publication costs of this article were defrayed in part by payment of page charges. This article must therefore be hereby marked "advertisement" in accordance with 18 USC section 1734 solely to indicate this fact. 


\section{References}

Bessoule, I.J., R. Lessire, and C. Cassagne. 1989. Partial purification of the acyl-CoA elongase of Allium porrum leaves. Arch. Biochem. Biophys. 268: 475-484.

Bianchi, G., C. Murelli, and E. Ottaviano. 1990. Maize pollen lipids. Phytochemistry 29: 739-744.

Bowman, J.L., D.R. Smyth, and E.M. Meyerowitz. 1991. Genetic interactions among floral homeotic genes of Arabidopsis. Development 112: 1-20.

Brewbaker, J.L. and B.H. Kwack. 1963. The essential role of calcium ion in pollen germination and tube growth. Am. I. Bot. 48: 457-464.

Dellaert, L.M.W., J.Y.P. Van Es, and M. Koornneef. 1979. Eceriferum mutants in Arabidopsis thaliana (L.) Heynh: Phenotypic and genetic analysis. Arabid. Inf. Serv. 16: 10-26.

Dickinson, H.G. and C.J. Elleman. 1985. Structural changes in the pollen grain of Brassica oleracea during dehydration in the anther and development on the stigma as revealed by anhydrous fixation techniques. Micron Microsc. Acta 16: 255-270.

Dickinson, H.G. and D. Lewis. 1973a. The formation of the tryphine coating the pollen grains of Raphanus, and its properties relating to the self-incompatibility system. Proc. $R$. Soc. Lond. B. Biol. Sci. 184: 149-165.

- 1973b. Cytochemical and ultrastructural differences between intraspecific pollinations in Raphanus. Proc. R. Soc. Lond. B Biol. Sci. 183: 21-38.

Dobson, H.E.M. 1989. Pollenkitt in plant reproduction. In The evolutionary ecology of plants (ed. J.H. Bock and Y.B. Linhart), pp. 227-246. Westview Press, Boulder, CO.

Dwyer, K.G., B.A. Lalonde, J.B. Nasrallah, and M.E. Nasrallah. 1992. Structure and expression of AtS1, an Arabidopsis thaliana gene homologous to the $S$-locus related genes of Brassica. Mol. Gen. Genet. 231: 442-448.

Elleman, C.J., V. Franklin-Tong, and H.G. Dickinson. 1992. Pollination in species with dry stigmas: the nature of the early stigmatic response and the pathway taken by pollen tubes. New Phytol. 121: 413-424.

Eschrich, W. and H.B. Currier. 1964. Identification of callose by its diachrome and fluorochrome reactions. Stain Technol. 39: 303-307.

Ferrari, T.E., P. Comstock, T.A. More, B. Best, S.S. Lee, and D.H. Wallace. 1983. Pollen-stigma interactions and intercellular recognition in Brassica. In Pollen: Biology and implications for plant breeding (ed. D.L. Mulcahy and E. Ottaviano), pp. 243-249. Elsevier, New York.

Hannoufa, A., J. McNevin, and B. Lemieux. 1993. Epicuticular wax of Arabidopsis thaliana eceriferum (cer) mutants. Phytochem. (in press.)

Heslop-Harrison, J. 1975. Incompatibility and the pollen-stigma interaction. Annu. Rev. Plant Physiol. 26: 403-425.

- 1979. Aspects of the structure, cytochemistry and germination of rye (Secale cereale). Ann. Bot. 44: 811-847.

Heslop-Harrison, J., R.B. Knox, and Y. Heslop-Harrison. 1974. Pollen wall proteins: Exine-held fractions associated with the incompatibility response in Cruciferae. Theor. Appl. Genet. 44: 133-137.

Hwang, I., T. Kohchi, B.M. Hauge, H.M. Goodman, R. Schmidt, G. Cnops, C. Dean, S. Gibson, K. Iba, B. Lemieux, V. Arondel, L. Danhoff, and C. Somerville. 1991. Identification and map position of YAC clones comprising one-third of the Arabidopsis genome. Plant I. 1: 367-374.

Kerhoas, C., R.B. Knox, and C. Dumas. 1983. Specificity of the callose response in stigmas of Brassica. Ann. Bot. 52: 597602.
Knox, R.B., M. Gaget, and C. Dumas. 1987. Mentor pollen techniques. Int. Rev. Cytol. 107: 315-332.

Koornneef, M. 1987. Linkage map of Arabidopsis thaliana $(2 \mathrm{n}=10)$. In Genetic maps 1987: A compilation of linkage and restriction maps of genetically studied organisms (ed. S.J. O'Brien), pp. 724-745. Cold Spring Harbor Laboratory, Cold Spring Harbor, New York.

Koornneef, M., C.J. Hanhart, and F. Thiel. 1989. A genetic and phenotypic description of Eceriferum (cer) mutants in Arabidopsis thaliana. J. Hered. 80: 118-122.

Lemieux, B., M. Miquel, C. Somerville, and J. Browse. 1990. Mutants of Arabidopsis with alterations in seed lipid fatty acid composition. Theor. Appl. Genet. 80: 234-240.

Mariani, C., M. De Beuckeleer, J. Truettner, J. Leemans, and R.B. Goldberg. 1990. Induction of male sterility in plants by a chimaeric ribonuclease gene. Nature 347: 737-741.

Mariani, C., V. Gossele, M. De Beuckeleer, M. De Block, R.B. Goldberg, W. De Greef, and J. Leemans. 1992. A chimaeric ribonuclease-inhibitor gene restores fertility to male sterile plants. Nature 357: 384-387.

Meyerowitz, E.M. 1989. Arabidopsis, a useful weed. Cell 56: 263-269.

Mo, Y., C. Nagel, and L.P. Taylor. 1992. Biochemical complementation of chalcone synthase mutants defines a role for flavonols in functional pollen. Proc. Natl. Acad. Sci. 89: 7213-7217.

Nasrallah, J.B., T. Nishio, and M.E. Nasrallah. 1991. The selfincompatibility genes of Brassica: Expression and use in genetic ablation of floral tissues. Annu. Rev. Plant Physiol. plant Mol. Biol. 42: 393-422.

Oliveira, L., A. Burns, T. Bisalputra, and K.-C. Yang. 1983. The use of an ultra-low viscosity medium (VCD/HXSA) in the rapid embedding of plant cells for electron microscopy. $I$. Microsc. 132: 195-202.

Roberts, I.N., A.D. Stead, D.J. Ockendon, and H.G. Dickinson. 1980. Pollen-stigma interactions in Brassica oleracea. Theor. Appl. Genet. 58: 241-246.

Roberts, I.N., G. Harrod, and H.G. Dickinson. 1984. Pollenstigma interactions in Brassica oleracea. J. Cell Sci. 66: 255264.

Sarker, R.H., C.J. Elleman, and H.G. Dickinson. 1988. Control of pollen hydration in Brassica requires continued protein synthesis, and glycosylation is necessary for intraspecific incompatibility. Proc. Natl. Acad. Sci. 85: 4340-4344.

Sarla, N. 1988. Overcoming interspecific incompatibility in the cross Brassica campestris ssp. japonica $\times$ Brassica oleracea var. botrytic using irradiated mentor pollen. Biol. Plant. (Prague) 30: 384-386.

Taylor, L.P. and R. Jorgensen. 1992. Conditional male fertility in chalcone synthase-deficient petunia. J. Hered. 83: 11-17.

Ylstra, B., A. Touraev, R.M.B. Moreno, E. Stoger, A.J. van Tunen, O. Vicente, I.N.M. Mol, and E. Heberle-Bors. 1992. Flavonols stimulate development, germination, and tube growth of tobacco pollen. Plant Physiol. 100: 902-907.

Zenkteler, M. 1990. In vitro fertilization and wide hybridization in higher plants. Crit. Rev. Plant Sci. 9: 267-279. 


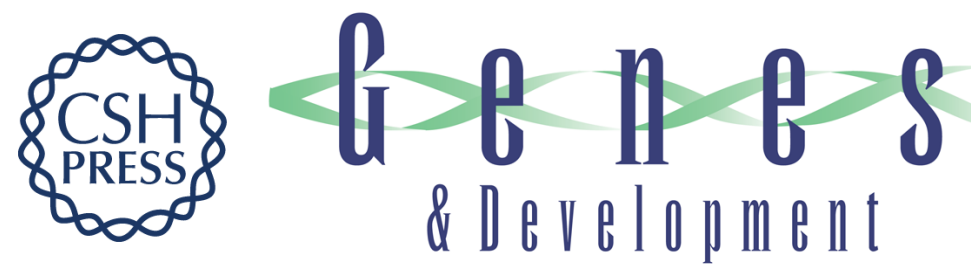

\section{A conditional sterile mutation eliminates surface components from Arabidopsis pollen and disrupts cell signaling during fertilization.}

D Preuss, B Lemieux, G Yen, et al.

Genes Dev. 1993, 7:

Access the most recent version at doi:10.1101/gad.7.6.974

References This article cites 32 articles, 5 of which can be accessed free at: http://genesdev.cshlp.org/content/7/6/974.full.html\#ref-list-1

License

Email Alerting

Service

Receive free email alerts when new articles cite this article - sign up in the box at the top right corner of the article or click here.

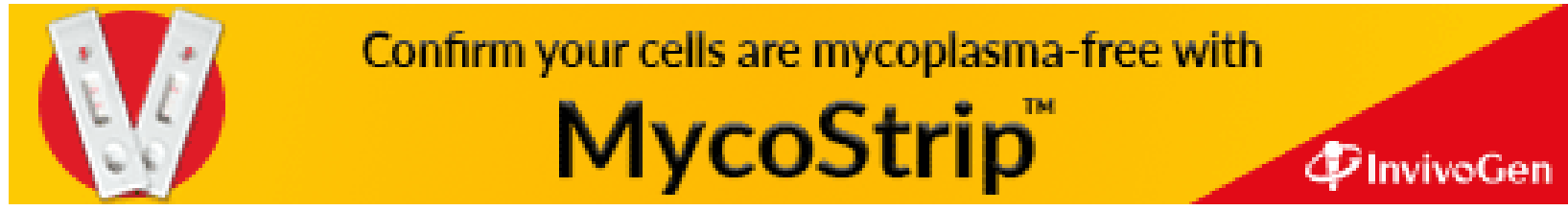

